\title{
BVRI Light Curves and Period Analysis of the Beta Lyrae System XX Leonis
}

\author{
P. Zasche ${ }^{1}$, P. Svoboda ${ }^{2}$, and M. Wolf ${ }^{1}$ \\ ${ }^{1}$ Astronomical Institute, Faculty of Mathematics and Physic, Charles University, \\ V Holešovičkách 2, CZ - 18000 Praha 8, Czech Republic, \\ email: petr.zasche@email.cz \\ ${ }^{2}$ Private observatory, Výpustky 5, CZ - 61400 Brno, Czech Republic
}

\begin{abstract}
The contact eclipsing binary system XX Leonis ( $p=0.97$ days, Sp A9) has been analysed using the PHOEBE programme, based on the Wilson-Devinney code. The $B V R I$ light curves were obtained during spring 2006 using the $20-\mathrm{cm}$ telescope and ST-7 CCD detector. The effective temperature of the primary component determined from the photometric analysis is $T=(7342 \pm 14) K$, the inclination of the orbit is $i=(84.83 \pm 0.29)^{\circ}$ and the photometric mass ratio $q=(0.40 \pm 0.01)$. The third-body hypothesis was also suggested; based on a period analysis using 57 minimum times this gave a period for the third body of $p_{3}=(59.66 \pm 0.05) \mathrm{yr}$, an amplitude of $A=(0.036 \pm 0.028)$ day and zero eccentricity, which yields as a minimum mass $M_{3, \min }=(0.91 \pm 0.01) M_{\odot}$.
\end{abstract}

Keywords. stars: binaries, binaries: close, binaries: eclipsing, stars: fundamental parameters, stars: individual (XX Leo)

\section{Introduction}

The binary system XX Leonis (SV* P 3370, AN 355.1934, BD+14 2177) was discovered to be a variable by Tsesevich (1954). It is an eclipsing binary star of $11^{\text {th }}$ magnitude, the period is close to one day and the depth of primary minimum is about 0.5 mag. The spectral type of the primary is A9V and of secondary K0. It was included in a catalogue of apsidal motion binaries by Hegedüs (1988), but this hypothesis was rejected.

\section{Light curve analysis}

The preliminary solution of the light curve (using the Washington filter system) computed by Shaw (1998) classified XX Leo as a near-contact binary, but the period analysis indicates constant period. A second analysis with satisfactory data was performed by Stark et al. (2000) but with only $R$ and $V$ light curves and also without a satisfactory light curve solution. So the masses of the components are still not very convincing. We assume here $M_{1}+M_{2}=2.41 M_{\odot}$, according to the derived spectral types from the previous analysis.

The new light elements for XX Leo derived from our period analysis using 57 minimum times (see Figure 2) are:

$$
\begin{gathered}
\text { Min I = HJD } 2450540.6831+0^{\mathrm{d}} .971135899 \cdot \mathrm{E} . \\
\pm 0.0076 \pm 0^{\mathrm{d}} .000000473
\end{gathered}
$$

We have measured the light curves using standard Bessell $B, V, R$, and $I$ filter system with a 20-cm telescope and an ST-7 CCD detector. These measurements were done at a private observatory in Brno, Czech Republic, during 20 nights in spring 2006. In Figure 1 

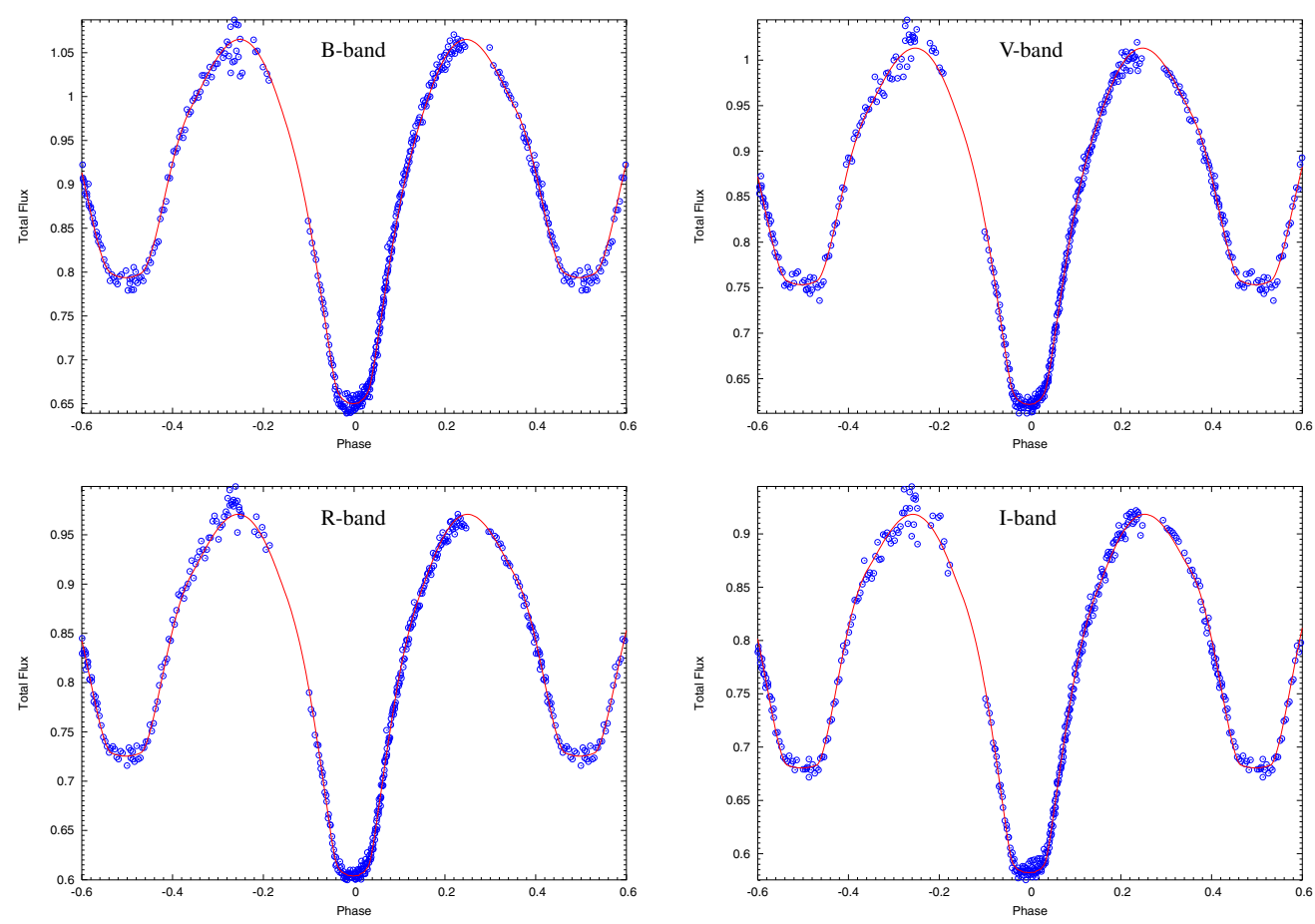

Figure 1. Light curves of XX Leo measured through Bessell filters $B, V, R$, and $I$ during spring 2006. Altogether 1495 data points (with no weightening scheme) and also the theoretical curves are plotted. On the $\mathrm{x}$-axis is the phase according to the ephemeris above and on the $\mathrm{y}$-axis is the relative total flux from the system.

you can see the theoretical curves with the individual data points. These measurements were analysed using the PHOEBE programme (see Prša 2005), based on the WilsonDevinney code (see, e.g., Wilson 1971). The final values for both components for the temperatures $\left(T_{i}\right)$, mass ratio $(q)$, inclination $(i)$, relative luminosities $\left(L_{1}, L_{2}, l_{3}\right)$, limbdarkening $\left(x_{i}\right.$, interpolated from the Van Hamme's tables), albedo $\left(A_{i}\right)$ and gravity darkening $\left(g_{i}\right)$ coefficients and synchronicity parameters $\left(F_{i}\right)$, respectively, are given in the Table 1. We have used the "double contact binary" mode during the computation, because it yields the best numerical result, the best agreement with previous results (primary and secondary spectral type A9 and K0, respectively) and also the period analysis. Regrettably we have no RV curve, so we cannot estimate other parameters, such as the masses and dimensions of the system, more precisely. The mass ratio computed only from the photometry is not very reliable. Spectroscopy of XX Leo would help us to estimate these parameters.

From the mass of the potential third body (about $0.9 M_{\odot}$, from the period analysis) we could conclude that this star on the main sequence should be of G9 spectral type. Also from the light curve analysis we can see that this body has almost the same luminosity as the secondary component, so both approaches lead to the same result. 


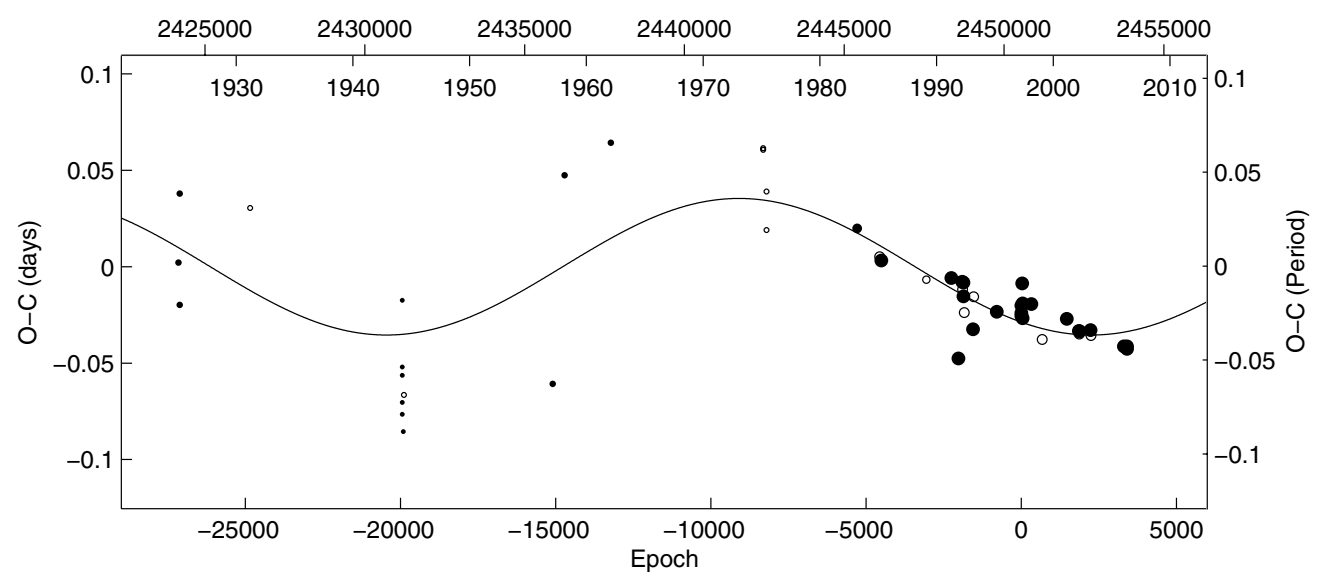

Figure 2. $O-C$ diagram of XX Leo.

\section{Period analysis}

The previous period analysis of XX Leo was performed by Stark et al. (2000), but no significant variation was found due to the small data set. Srivastava (1994) also found that the period of the binary is constant. We have used 57 minimum times covering more than 80 years. In Figure 2, individual minimum times are marked as dots and circles for the primary and secondary minimum, respectively. We see no evident displacement of secondary minima, so the apsidal motion could be ruled out. Symbols are scaled with the weight. The light-time effect hypothesis curve is also shown. The final results of the fit are in Table 2. We have fixed the value of eccentricity to zero because of the best agreement with the photometric results.

From the third-body hypothesis the mass function and minimum mass of the third body were also calculated. The mass function is $f\left(m_{3}\right)=0.068 M_{\odot}$, so the minimum mass of the third body is $0.91 \pm 0.02 M_{\odot}$. As we can see, this suggested body might be a bit more massive than the secondary component, which is in good agreement with the luminosities of the individual components found in the light curve analysis. The secondary

Table 1. Physical parameters of XX Leo.

\begin{tabular}{cccc}
\hline Parameter & Value & Parameter & Value \\
\hline$T_{1}[K]$ & $7342 \pm 14$ & $x_{1, B}$ & $0.603 \pm 0.009$ \\
$T_{2}[K]$ & $4991 \pm 88$ & $x_{1, V}$ & $0.508 \pm 0.009$ \\
$M_{2} / M_{1}$ & $0.401 \pm 0.002$ & $x_{1, R}$ & $0.408 \pm 0.008$ \\
$i\left[^{\circ}\right]$ & $84.835 \pm 0.287$ & $x_{1, I}$ & $0.320 \pm 0.010$ \\
$L_{1, B}$ & $0.891 \pm 0.003$ & $x_{2, B}$ & $0.866 \pm 0.115$ \\
$L_{1, V}$ & $0.841 \pm 0.002$ & $x_{2, V}$ & $0.724 \pm 0.095$ \\
$L_{1, R}$ & $0.809 \pm 0.002$ & $x_{2, R}$ & $0.597 \pm 0.079$ \\
$L_{1, I}$ & $0.783 \pm 0.002$ & $x_{2, I}$ & $0.489 \pm 0.080$ \\
$L_{2, B}$ & $0.070 \pm 0.002$ & $A_{1}$ & $0.215 \pm 0.019$ \\
$L_{2, V}$ & $0.079 \pm 0.002$ & $A_{2}$ & $0.875 \pm 0.094$ \\
$L_{2, R}$ & $0.089 \pm 0.001$ & $g_{1}$ & $0.807 \pm 0.034$ \\
$L_{2, I}$ & $0.106 \pm 0.001$ & $g_{2}$ & $0.069 \pm 0.087$ \\
$l_{3, B}$ & $0.040 \pm 0.003$ & $F_{1}$ & $0($ fixed $)$ \\
$l_{3, V}$ & $0.080 \pm 0.002$ & $F_{2}$ & $2.249 \pm 0.018$ \\
$l_{3, R}$ & $0.102 \pm 0.002$ & & \\
$l_{3, I}$ & $0.111 \pm 0.002$ & & \\
\hline
\end{tabular}


Table 2. Parameters of the third component.

\begin{tabular}{cc}
\hline Parameter & Value \\
\hline$p_{3}(\mathrm{yr})$ & $59.66 \pm 0.05$ \\
$A(\mathrm{~d})$ & $0.036 \pm 0.028$ \\
$\omega\left[^{\circ}\right]$ & $19.22 \pm 12.98$ \\
$e$ & 0 (fixed) \\
$T_{0}(\mathrm{JD})$ & $2437200.62 \pm 216.63$ \\
\hline
\end{tabular}

and tertiary components seem to be rather similar, what could be an interesting example for spectral disentangling.

\section{Conclusions}

We have performed new analysis of the $\beta$ Lyrae-type contact binary XX Leo. New light curves were measured and analysed, and a new period study has been done. The hypothesis of the third body was suggested, according to the third light in the LC solution and also from the light-time effect hypothesis. Both hypothesis are in very good agreement and leads to a third body comparable to the secondary.

\section{Acknowledgements}

This research has made use of the SIMBAD database, operated at CDS, Strasbourg, France, and of NASA's Astrophysics Data System Bibliographic Services. This investigation was supported by the Grant Agency of the Czech Republic, grant No. 205/04/2063 and No. 205/06/0217.

\section{References}

Hegedüs, T. 1988, BICDS 35, 15

Prša, A. \& Zwitter,T. 2005, AJ 628, 426

Shaw, J.S. \& Nicol, S. 1998, IAPPP Comm 71, 57

Srivastava, R.K. 1994, ApESSS 219, 291

Stark, M.A., et al. 2000, JAVSO 28, 25

Tsesevich, V.P. 1954, Odd.Izv. 4, 2

Wilson, R.E. \& Devinney, E. J. 1971, ApJ 166, 605 